\title{
Adenocarcinoma adjacent to a rare anatomical variant of right upper lobe
}

\author{
Cheng Shen, Guowei Che \\ Department of Thoracic Surgery, West-China Hospital, Sichuan University, Chengdu, China
}

Kardiochirurgia i Torakochirurgia Polska 2018; 15 (2): 141-142

We read with great interest the paper by Samancilar et al. [1], accepted for publication in "Kardiochirurgia i Torakochirurgia Polska/Polish Journal of Thoracic and Cardiovascular Surgery". The authors report the case of a patient with an azygos lobe who underwent videothoracoscopic lung resection due to the presence of non-small-cell lung carcinoma in the upper lobe of the right lung. We present a new case with typical figures in surgery to facilitate the preoperative diagnosis and avoid the misdiagnosis of such a rare disease.

A 51-year-old woman was referred to our hospital for assessment of ground-glass opacity (GGO) that was detected on chest radiography during a routine health check. Physical examination revealed normal breathing sounds in both lung fields. Laboratory findings were within normal limits. Her pulmonary function tests and cardiovascular examination revealed normal performance. High-resolution chest computed tomography (HRCT) scans confirmed presence of an azygos lobe and a GGO measuring $1.2 \times 1.0 \mathrm{~cm}$ in the anterior segment of the right upper lobe adjacent to the arch of the azygos vein (Figs. 1 A, B). Bronchoscopy was normal and bronchoalveolar lavage from the right upper lobe was negative for malignancy. As diagnosis of the nodule was not established through imaging and bronchoscopic examination, surgery was scheduled. We approached the GGO with mediastinal lymph node dissection; it was removed via video-assisted thoracic surgery (VATS). The azygos lobe was exposed during the operation. The upper part of the pleural cavity was seen to be divided into two compartments by a dome-like fold and occupied the fissure. The fold is a reduplication of the parietal pleura. Its convex margin is attached along a line on the thoracic wall; the attachment commences posteriorly at the right side of the fifth thoracic vertebra, passes obliquely upwards across the posterior parts of the intercostal spaces to the middle of the second rib, and then downwards and forwards to the first costal cartilage, where it disappears in the parietal pleura of the front of the thorax. The free margin of the fold is concave and contains between its two layers the azygos vein (Fig. 2 A). Frozen section of the specimen revealed a well-differentiated adenocarcinoma with negative tumor margins (Fig. 2 B). The patient was discharged 3 days after surgery without major complications. Furthermore, recurrence was not observed during the 1-year follow-up period.
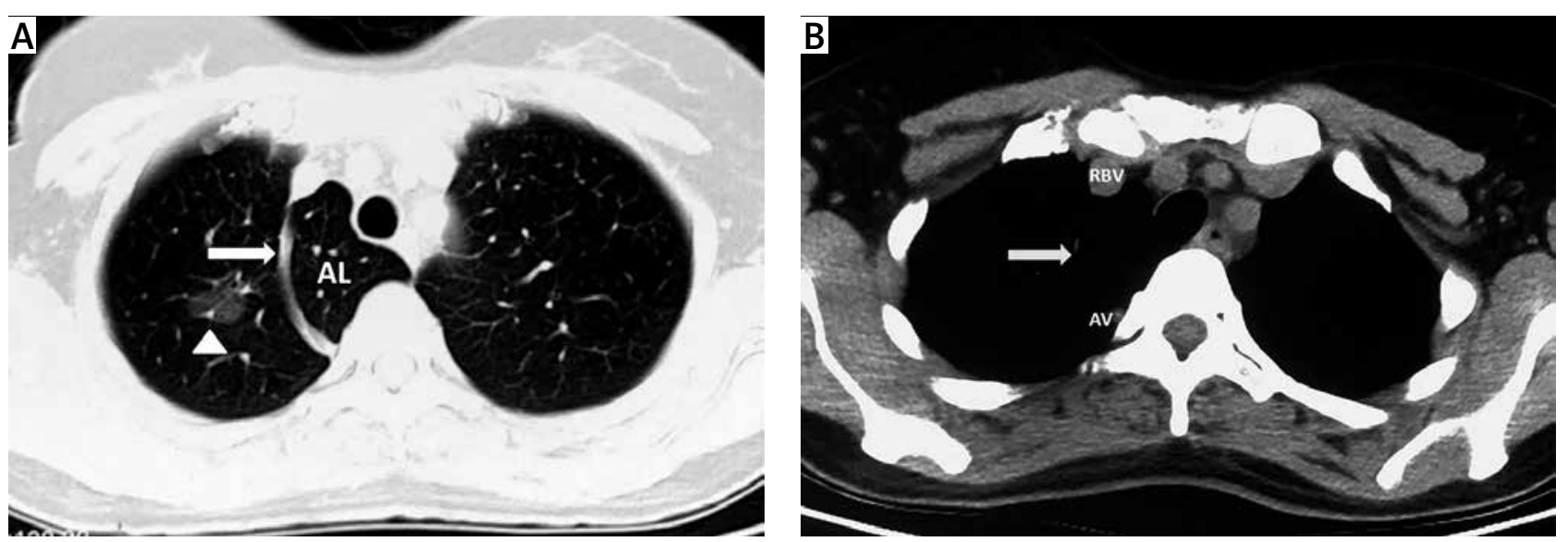

Fig. 1. Computed tomography features of the case. A - Chest HRCT showing azygos lobe (AL) and azygos vein (arrow); GGO in right upper lobe adjacent to azygos lobe (arrowhead). B - Chest HRCT also showing the arch of the azygos vein (arrow) between the azygos vein (AV) and right brachial vein (RBV)

Address for correspondence: Guowei Che MD, PhD, Department of Thoracic Surgery, West-China Hospital, Sichuan University, Guoxuexiang 37, 610041 Chengdu, China, phone: +86 28 85422494, fax: +86 28 85422493, e-mail: cheguowei_hx@aliyun.com Received: 23.01.2018, accepted: 17.02.2018. 

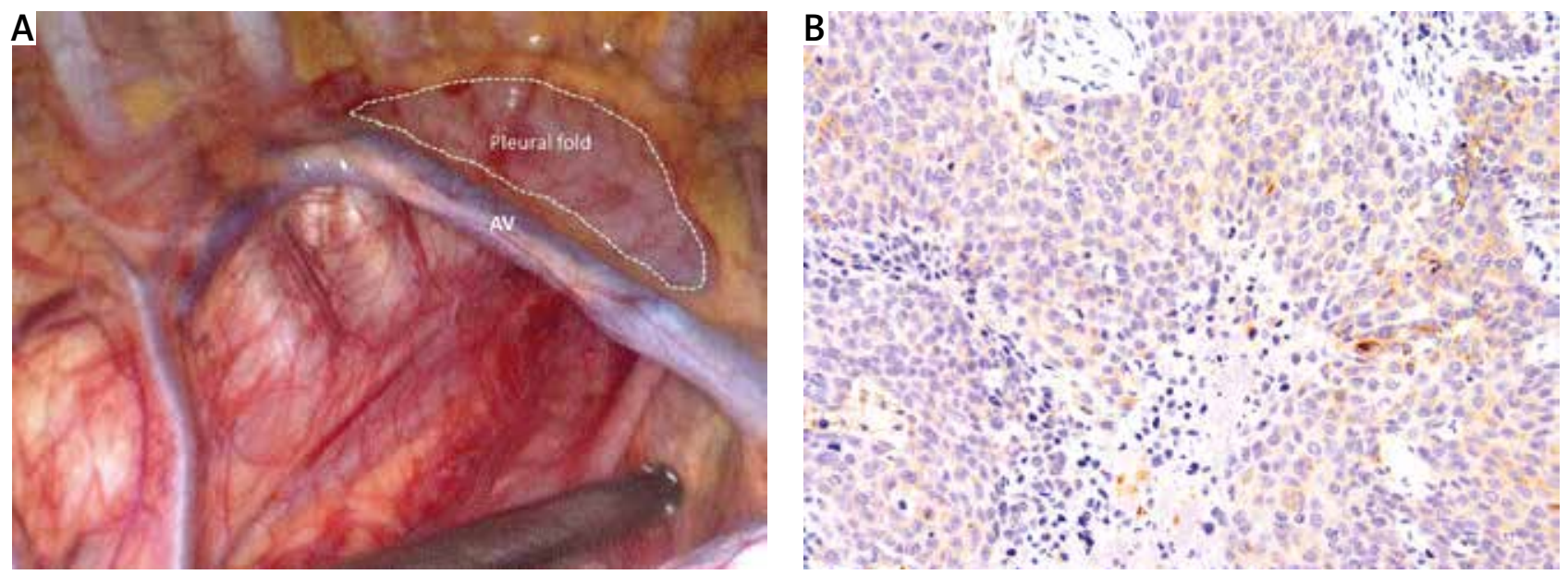

Fig. 2. Intraoperative image of the azygos lobe and histological and immunohistochemical characteristics of lesion. A - A dome-like fold located in the upper right lobe. Its convex margin commences posteriorly at the right side of the fifth thoracic vertebra and then downwards and forwards to the first costal cartilage, where it disappears in the parietal pleura of the front of the thorax. The free margin of the fold is concave and contains between its two layers the azygos vein (AV). B - Microscopic image of adenocarcinoma (200x)

An azygos lobe is a congenital variation in the apex of the right lung in $0.11-1.06 \%$ of the population and it was first described by Heinrich Wrisberg in 1877 [2]. Some tumors may originate directly from the azygos lobe, and studies on the subject are rare [3]. An azygos lobe is not a true accessory lobe as it does not have its own bronchus, it does not correspond to a specific bronchopulmonary segment, and it is formed due to penetration of the right posterior cardinal vein, one of the precursors of the azygos vein, into the apex of the lung instead of normal migration over it during embryogenesis [4].

\section{Disclosure}

Authors report no conflict of interest.

\section{References}

1. Samancilar O, Akçam Ti, Kaya SO, Sevinc S, Akcay O, Ceylan KC. Video-assisted thoracoscopic surgery (VATS) right upper lobectomy for non-small-cell lung cancer with an azygos lobe. Kardiochir Torakochir Pol 2016; 13: 373-4.

2. Karre PR, Cooper GB II. The azygos lobe and vein: interesting and typical clinical image. BMJ Case Rep 2011; 2011. doi: 10.1136/bcr.05.2011.4266.

3. Shakir HA. Removal of aberrant azygos lobe containing positron emission tomography positive nodule with the use of video-assisted thoracic surgery. Int J Surg Case Rep 2014; 5: 95-6.

4. Pradhan G, Sahoo S, Mohankudo S, Dhanurdhar Y, Jagaty SK. Azygos lobe a rare anatomical variant. J Clin Diagn Res 2017; 11: TJ02. 